\title{
Instability of sputum molecular phenotypes in U-BIOPRED severe asthma
}

\author{
To the Editor:
}

The Unbiased Biomarkers for the Prediction of Respiratory Disease Outcomes (U-BIOPRED) project has described phenotypic differences of severe asthma using a systems biology approach. We obtained three molecular phenotypes termed transcription-associated clusters (TACs) using hierarchical clustering of differentially expressed transcripts between T2-high and T2-low [1]. TAC1 was characterised by receptors IL33R, CCR3 and TSLPR, with the highest enrichment of gene signatures for IL-13/type-2 (T2) inflammation with sputum eosinophilia; TAC2 by inflammasome-associated genes, interferon- $\alpha$ (IFN- $\alpha$ ) and tumour necrosis factor- $\alpha$ (TNF- $\alpha$ )-associated genes with sputum neutrophilia; and TAC3 by metabolic and mitochondrial function genes with pauci-granulocytic inflammation. Given that sputum eosinophilia may vary with time in many asthmatic subjects $[2,3]$, we hypothesised that TAC status may also change with time.

Of 421 U-BIOPRED subjects with severe asthma at the baseline visit, 321 returned for a second visit at 1 year and whole sputum samples were obtained by induction with hypertonic saline for differential cell count and for transcriptomic analysis at both visits. All visits were made at a time when the subjects had been free of an exacerbation in the past 4 weeks. Expression profiling was performed using Affymetrix U133 Plus 2.0 (Affymetrix, Santa Clara, CA, USA) microarray with RNA extracted from sputum cells. In 38 patients who returned for the second visit, we obtained good quality transcriptomic data from sputum cells [4]. Hierarchical clustering based on Euclidean distance was performed on the transcriptomic data using the reduced 77 gene-set that defined the original three TACs [1]. This led to the definition of the same three TACs as previously defined with the characteristics of each TAC being preserved. The distribution of sputum eosinophils (EOS) and sputum neutrophils (NEU) counts (reported as mean with 25-75\% interquartile range and number) between the three TACs remained unchanged compared to that found at their first visit (TAC1, first visit: 44\% (24.2-53.4\%, n=6) EOS and 45.1\% (30.2-65.3\%, n=6) NEU, and second visit: $28 \%(15.3-44.5 \%, \mathrm{n}=12)$ EOS and 41.6\% (31.7-65.1\%, $\mathrm{n}=12)$ NEU; TAC2, first visit: 1.4\% (0.5-5.0\%, $\mathrm{n}=16)$ EOS and 78.2\% (65.9-84.8\%, $\mathrm{n}=16)$ NEU, and second visit: $2 \%(0.3-2.8 \%, \mathrm{n}=8)$ EOS and 92.3\% (84.9-94.3, n=8) NEU; TAC3, first visit: $2.7 \%(1.1-10.8 \%, \mathrm{n}=16)$ EOS and 52.3\% (40.5$59.4 \%, \mathrm{n}=16) \mathrm{NEU}$, and second visit: $1.5 \%(0.2-3.9 \%, \mathrm{n}=18)$ EOS and 50.6\% (44.5-62.4\%, $\mathrm{n}=18) \mathrm{NEU})$. Thus, the pattern of sputum granulocytic-defined inflammation within the TACs remained unchanged (figure 1a). Therefore, for the whole group, there was no significant differences in sputum granulocytic composition between baseline and the 1-year follow-up.

However, when the data was analysed in terms of individual shifts of TAC between the first and the second visit, as shown on the Sankey flow plot which shows the movement of the granulocytic inflammatory status in relation to the TAC cluster between the baseline and follow-up visit, with the width of the flow proportional to the number of subjects (figure 1b), 21 out of 38 patients remained in the same TAC at 1 year (five of 12 in TAC1, five of eight in TAC2 and 11 of 18 in TAC3). In the remainder, seven TAC1 patients changed to TAC2 $(n=4,33 \%$ of baseline TAC1) or TAC3 $(n=3,25 \%$ of baseline TAC1), three TAC2 subjects moved to either TAC1 $(n=1,12 \%$ of baseline TAC2) or TAC3 $(n=2,25 \%$ of baseline TAC2), while seven TAC3 subjects changed to TAC2 (61\% of baseline TAC3). The kappa statistic was 0.24 (95\% CI -0.08-0.56), indicating a fair to minimal agreement between TACs at baseline compared to follow-up TACs for matched samples.

@ERSpublications

At 1 year, $45 \%$ of severe asthma change molecular phenotype as determined by sputum transcriptomic analysis. Together with concomitant shift in sputum granulocytic markers, this may indicate variability of driving mechanisms in this unstable group. https://bit.ly/35aj489

Cite this article as: Kermani NZ, Pavlidis S, Xie J, et al. Instability of sputum molecular phenotypes in UBIOPRED severe asthma. Eur Respir J 2021; 57: 2001836 [https://doi.org/10.1183/13993003.01836-2020]. 


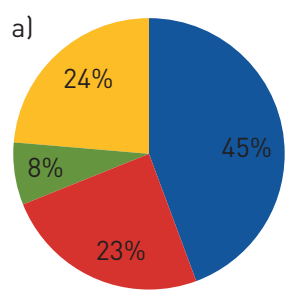

Eosinophillic Mixed

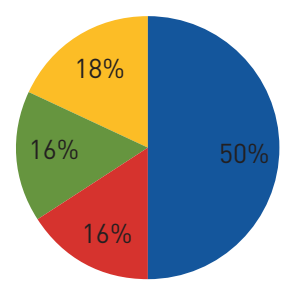

Neutrophillic

- PauciGranulocitic b)

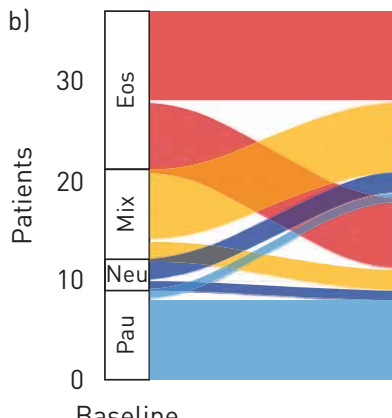

Baseline inflammatory phenotypes

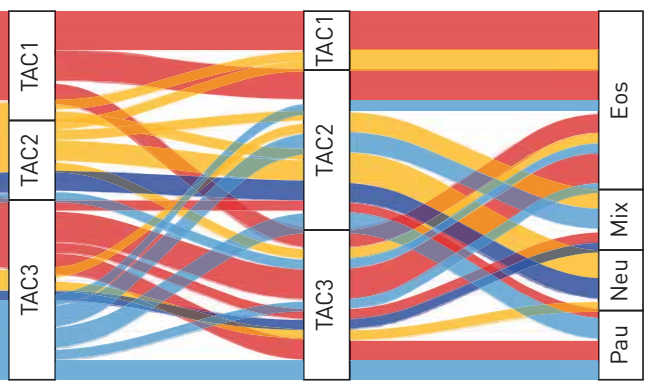

Follow-up TACs
Follow-up inflammatory phenotypes c)

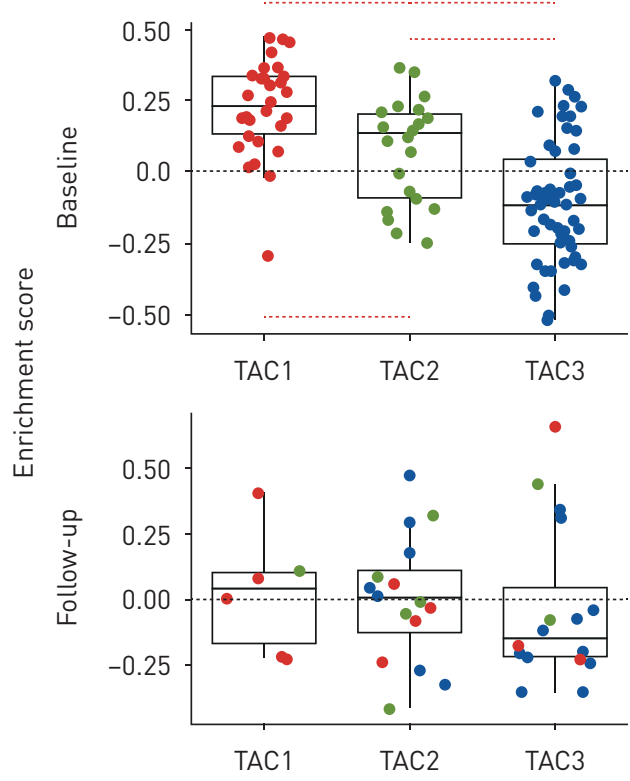

Ageing

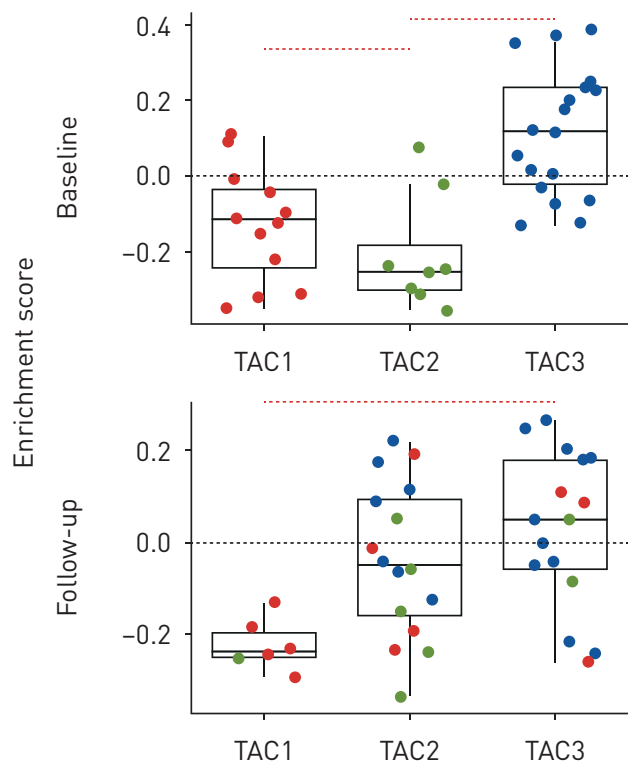

Inflammasome signature
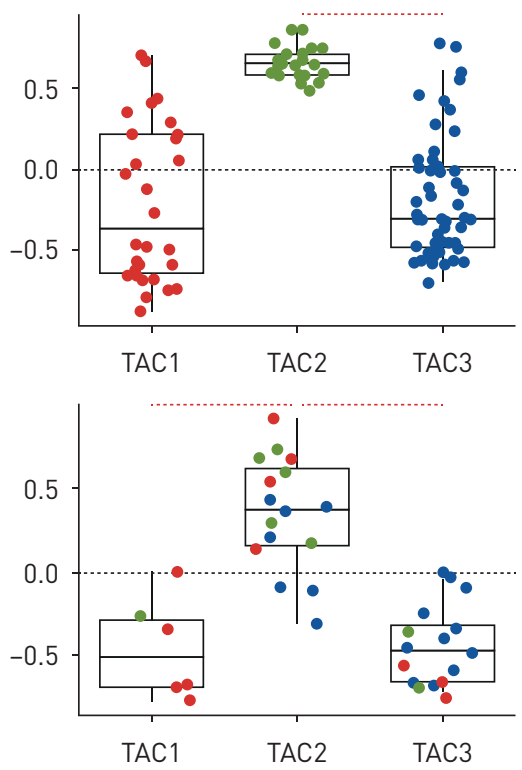

Neutrophil activation
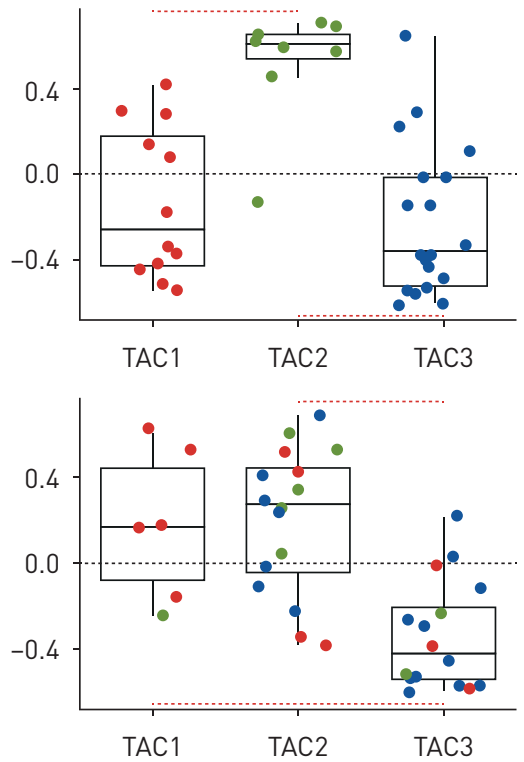

OXPHOS
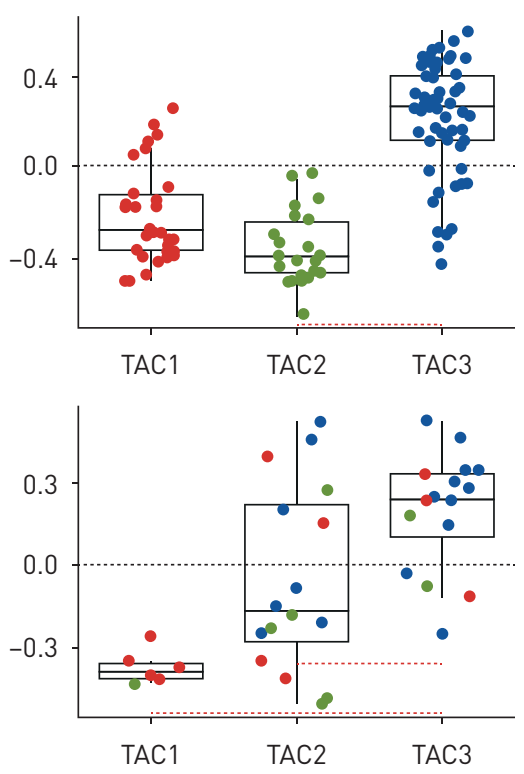

ILC2
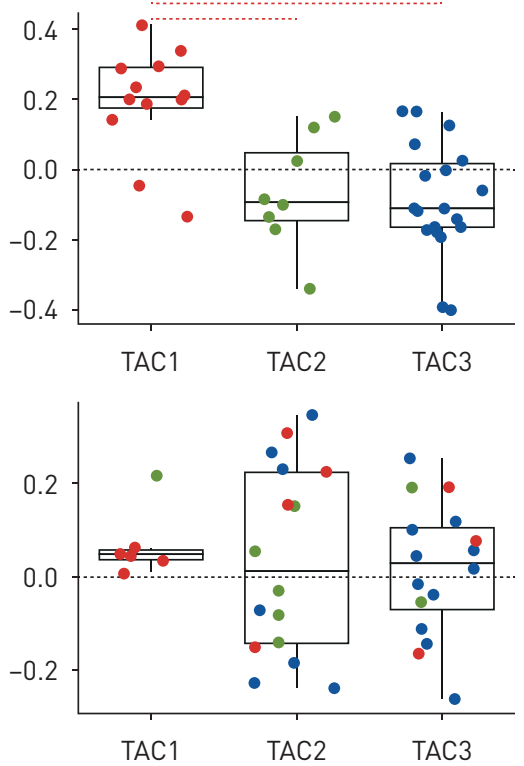
FIGURE 1 a) Pie charts showing the distribution of granulocytic inflammation measured in sputum cells in 38 patients with severe asthma at first visit and at follow-up at 1 year. Eosinophilia defined as eosinophil count $\geqslant 1.5 \%$; neutrophilia as neutrophil count $\geqslant 74 \%$; mixed as neutrophilic and eosinophilic; and paucigranulocytic as neither neutrophilic nor eosinophilic. b) Sankey plot showing the flow of transcription-associated cluster (TAC) membership and sputum granulocyte inflammation at baseline and at 1 year. Eos: eosinophilic; neu: neutrophilic; mix: mixed eosinophilic and neutrophilic; pauci: paucigranulocytic. c) Dot plot relative enrichment scores with box and whisker plots showing median and interquartile range for six pathway signatures assessed at baseline (upper panels), and at 1 year follow-up (lower panels) on sputum transcriptomics using gene set variation analysis. Patient samples are colour-based according to baseline cluster membership in the TACs. ILC2: innate lymphoid cell type 2; IL-13/Th2: interleukin-13/T-helper type 2; OXPHOS: oxidative phosphorylation.

We determined whether there were any characteristics measured at the first visit that could distinguish those that remained stable and those that changed TAC status within each of the three TAC classes. There were no significant differences in blood or sputum markers of granulocytic inflammation within each TAC shift between those that remained stable and those that moved. For those that shifted from TAC1, there was less allergic rhinitis $(p<0.001)$ and eczema $(p=0.0005)$, but no differences in inflammatory markers compared to the stable TAC1 group. For those that changed from TAC2 compared to stable TAC2, they were non-smokers $(\mathrm{p}<0.001)$ and had less nasal polyps $(\mathrm{p}<0.001)$ and eczema $(\mathrm{p}<0.001)$, but more were on oral corticosteroid therapy $(\mathrm{p}<0.001)$ with higher total serum $\operatorname{IgE}(\mathrm{p}=0.05)$ and a less likelihood of a previous history of pneumonia. For those that changed from TAC3, they had more allergic rhinitis $(\mathrm{p}<0.001)$, more eczema $(\mathrm{p}<0.001)$, more oral corticosteroid usage $(\mathrm{p}<0.001)$ and less history of pneumonia $(p<0.01)$ compared to stable TAC3. Therefore, there may be factors that determine the molecular instability of each of the three TACs over the 1 year period.

We used gene set variation analysis (GSVA) to determine the relative expression scores of specific pathways [5] in relation to TAC status using signatures indicative of IL-13-Th2 [6], innate lymphoid type 2 (ILC2) [7], neutrophil [8], and inflammasome activation [9], and oxidative phosphorylation [10] and senescence signatures [11]. We confirmed that TAC2 subjects continued to have the highest expression score for neutrophil and inflammasome activation and TAC3 subjects for OXPHOS and ageing signatures (figure 1c) [1], similar to that described at the baseline visit. However, the IL13/Th2 and ILC2 signature enrichment were reduced in TAC1 subjects at follow-up. Thus, GSVA indicates that with the shift in TAC assignment, the relative importance of certain specific pathways characterising each TAC has changed from baseline.

Although only representative of a small proportion of the original U-BIOPRED severe asthma cohort, we have shown that the molecular phenotypes of severe asthma derived from an analysis of the sputum transcriptome can be unstable at 1 year in nearly half of the patients. TAC assignment was stable in the majority of patients $(55 \%)$ at 1 year, but in $45 \%$ of the patients, there was a change in the TAC status, mainly from TAC1 or TAC3 to TAC2 status. In the seven subjects who were in TAC1 and who changed into TAC2 or TAC3, the sputum eosinophil count fell from $25.2 \%$ to $16.3 \%(\mathrm{p}<0.003)$, indicating that this shift in TAC may be determined by factors that influence eosinophilic inflammation, such as adherence to or use of corticosteroid treatment. Within the TAC2 or TAC3 subjects at baseline, those who were on oral corticosteroids were more likely to switch to other TAC categories. There were no significant differences in asthma therapy between those who remain stable and those who changed TAC status in terms of antibiotic, biologic (anti-IgE antibody) and bursts of systemic corticosteroid use.

The major drawback in our study is the relatively small numbers of subjects studied cross-sectionally. A much larger longitudinal study with a larger number of follow-up patients done at multiple time-points is needed to confirm these findings of instability of transcriptomic clusters. However, this study supports the report of instability of clinical clusters based on clinical, physiologic and biomarker inflammatory data over time [12-15]. An unbiased cluster analysis of exhaled metabolomic fingerprint in 78 patients with severe asthma of the U-BIOPRED cohort led to definition of three distinct clusters, and the follow-up clustering at 1 year also showed that $41 \%$ of the cluster was stable while $59 \%$ moved to the other two clusters [16]. As shown here, these shifts in phenotype were accompanied by appropriate shifts in granulocytic inflammation. Thus, although an asthmatic subject may change TAC status, the composition of each TAC class remains stable with similar inflammatory and transcript profiles. The mechanism(s) driving the molecular switch in some patients remains uncertain from this study, but this may involve the presence of sub-clinical infection, defective resolving mechanisms or perhaps changes in environmental or chronic treatment conditions. Our limited amount of data indicates that patient factors, such as the presence of comorbidities and use of oral corticosteroid therapy, may potentially determine the stability of the TAC status. We did not find that the instability was associated with frequent exacerbations. The instability of TACs indicate that targeted biologic therapies that block type- 2 inflammation that would be appropriate for TAC1 phenotype may become less effective in those that shift from TAC1 to TAC2 or TAC3. 
Nazanin Z. Kermani ${ }^{1}$, Stelios Pavlidis ${ }^{1,2}$, Jiaxing $\mathrm{Xie}^{2}$, Kai Sun ${ }^{1}$, Matthew Loza ${ }^{3}$, Fred Baribaud ${ }^{3}$, Steve J. Fowler $\oplus^{4}$, Dominic E. Shaw $\oplus^{5}$, Louise J. Fleming $\oplus^{2}$, Peter H. Howarth ${ }^{6}$, Ana R. Sousa ${ }^{7}$, Julie Corfield ${ }^{8,9}$, Charles Auffray ${ }^{10}$, Bertrand De Meulder $^{10}$, Peter J. Sterk ${ }^{11}$, Yike Guo ${ }^{1}$, Mohib Uddin ${ }^{12}$, Ratko Djukanovic ${ }^{6}$, Ian M. Adcock $\oplus^{1,2}$ and Kian Fan Chung ${ }^{1,2}$ on behalf of the U-BIOPRED study group

${ }^{1}$ Dept of Computing and Data Science Institute, Imperial College London, London, UK. ${ }^{2}$ National Heart and Lung Institute, Imperial College London, and Biomedical Research Unit, Royal Brompton and Harefield NHS Trust, London, UK. ${ }^{3}$ Janssen Research and Development, High Wycombe, UK. ${ }^{4}$ Centre for Respiratory Medicine and Allergy, Institute of Inflammation and Repair, University of Manchester and University Hospital of South Manchester, Manchester, UK. ${ }^{5}$ Respiratory Research Unit, University of Nottingham, Nottingham, UK. ${ }^{6}$ NIHR Southampton Respiratory Biomedical Research Unit, Clinical and Experimental Sciences and Human Development and Health, Southampton, UK. ${ }^{7}$ Respiratory Therapeutic Unit, GSK, Stockley Park, UK. ${ }^{8}$ AstraZeneca R\&D, Molndal, Sweden. ${ }^{9}$ Areteva R\&D, Nottingham, UK. ${ }^{10}$ European Institute for Systems Biology and Medicine, CNRS-ENS-UCBL-INSERM, Lyon, France. ${ }^{11}$ Amsterdam UMC, University of Amsterdam, Amsterdam, The Netherlands. ${ }^{12}$ Respiratory Global Medicines Development, AstraZeneca, Gothenburg, Sweden.

Correspondence: Kian Fan Chung, National Heart and Lung Institute, Imperial College London, Dovehouse Street, London SW3 6LY, UK. E-mail: f.chung@imperial.ac.uk

Received: 17 May 2020 | Accepted: 27 Aug 2020

Conflict of interest: N.Z. Kermani has nothing to disclose. S. Pavlidis has nothing to disclose. J. Xie has nothing to disclose. K. Sun has nothing to disclose. M. Loza is an employee and shareholder of Janssen R\&D, Pharmaceutical Companies of Johnson \& Johnson. F. Baribaud is an employee and shareholder of Janssen R\&D, Pharmaceutical Companies of Johnson \& Johnson. S.J. Fowler reports grants from Innovative Medicines Initiative (IMI), during the conduct of the study; personal fees from GlaxoSmithKline, AstraZeneca, Boehringer Ingelheim and Novartis, outside the submitted work. D.E. Shaw reports personal fees from GSK, AstraZeneca, Teva and Boehringer Ingelheim, outside the submitted work. L.J. Fleming reports personal fees for advisory board membership from Novartis, Vectura and Boehringer Ingelheim; grants for research from Asthma UK and BLF; personal fees for lectures from Novartis, outside the submitted work. P.H. Howarth reports part time employment by GSK as Global Medical Expert. A.R. Sousa has nothing to disclose. J. Corfield has nothing to disclose. C. Auffray has nothing to disclose. B. De Muelder has nothing to disclose. P.J. Sterk reports grants from Innovative Medicines Initiative (IMI), during the conduct of the study. Y. Guo has nothing to disclose. M. Uddin is an employee of AstraZeneca and holds shares in the company. R. Djukanovic reports personal fees from TEVA, grants and personal fees from Novartis, and personal fees and other support from Synairgen outside the submitted work. I.M. Adcock reports personal fees for advisory board membership from GSK, AstraZeneca, Novartis, Boehringer Ingelehim and Vectura; grants from Pfizer, GSK, MRC, EU, Boehringer Ingelheim and Innovative Medicines Initiative (IMI); and personal fees for lectures, outside the submitted work. K.F. Chung has received honoraria for participating on advisory board meetings of GSK, AstraZeneca, Novartis, Merck, Boehringer Ingelheim, Roche and TEVA regarding treatments for asthma and COPD and on the scientific advisory board of the Clean Breathing Institute supported by GSK Health Care Consumer Products; and has also been remunerated for speaking engagements by AstraZeneca, Novartis and Merck, outside the submitted work.

Support statement: The U-BIOPRED project is supported through an Innovative Medicines Initiative Joint Undertaking under grant agreement 115010, resources of which are composed of financial contributions from the European Union's Seventh Framework Programme (FP7/2007-2013) and European Federation of Pharmaceutical Industries and Associations companies' in-kind contributions (www.imi.europa.eu). Funding information for this article has been deposited with the Crossref Funder Registry.

\section{References}

1 Kuo CS, Pavlidis S, Loza M, et al. T-helper cell type 2 (Th2) and non-Th2 molecular phenotypes of asthma using sputum transcriptomics in U-BIOPRED. Eur Respir J 2017; 49: 1602135.

2 Al-Samri MT, Benedetti A, Prefontaine D, et al. Variability of sputum inflammatory cells in asthmatic patients receiving corticosteroid therapy: a prospective study using multiple samples. J Allergy Clin Immunol 2010; 125: $1161-3$ e4.

3 McGrath KW, Icitovic N, Boushey HA, et al. A large subgroup of mild-to-moderate asthma is persistently noneosinophilic. Am J Respir Crit Care Med 2012; 185: 612-619.

4 Rossios C, Pavlidis S, Hoda U, et al. Sputum transcriptomics reveal upregulation of IL-1 receptor family members in patients with severe asthma. J Allergy Clin Immunol 2018; 141: 560-570.

5 Pavlidis S, Monast C, Loza MJ, et al. I_MDS: an inflammatory bowel disease molecular activity score to classify patients with differing disease-driving pathways and therapeutic response to anti-TNF treatment. PLoS Comput Biol 2019; 15: e1006951.

6 Alevy YG, Patel AC, Romero AG, et al. IL-13-induced airway mucus production is attenuated by MAPK13 inhibition. J Clin Invest 2012; 122: 4555-4568.

7 Bjorklund AK, Forkel M, Picelli S, et al. The heterogeneity of human CD127(+) innate lymphoid cells revealed by single-cell RNA sequencing. Nat Immunol 2016; 17: 451-460.

8 Abbas AR, Baldwin D, Ma Y, et al. Immune response in silico (IRIS): immune-specific genes identified from a compendium of microarray expression data. Genes Immun 2005; 6: 319-331.

9 Simpson JL, Phipps S, Baines KJ, et al. Elevated expression of the NLRP3 inflammasome in neutrophilic asthma. Eur Respir J 2014; 43: 1067-1076.

10 Subramanian A, Tamayo P, Mootha VK, et al. Gene set enrichment analysis: a knowledge-based approach for interpreting genome-wide expression profiles. Proc Natl Acad Sci USA 2005; 102: 15545-15550.

11 de Magalhaes JP, Curado J, Church GM. Meta-analysis of age-related gene expression profiles identifies common signatures of aging. Bioinformatics 2009; 25: 875-881.

12 Newby C, Heaney LG, Menzies-Gow A, et al. Statistical cluster analysis of the British Thoracic Society Severe refractory Asthma Registry: clinical outcomes and phenotype stability. PLoS One 2014; 9: e102987. 
13 Zaihra T, Walsh CJ, Ahmed S, et al. Phenotyping of difficult asthma using longitudinal physiological and biomarker measurements reveals significant differences in stability between clusters. BMC Pulm Med 2016; 16: 74.

14 Kupczyk M, Dahlen B, Sterk PJ, et al. Stability of phenotypes defined by physiological variables and biomarkers in adults with asthma. Allergy 2014; 69: 1198-1204.

15 Loza MJ, Djukanovic R, Chung KF, et al. Validated and longitudinally stable asthma phenotypes based on cluster analysis of the ADEPT study. Respir Res 2016; 17: 165.

16 Brinkman $\mathrm{P}$, Wagener AH, Hekking PP, et al. Identification and prospective stability of electronic nose (eNose)-derived inflammatory phenotypes in patients with severe asthma. J Allergy Clin Immunol 2018; 74: 406-411. 